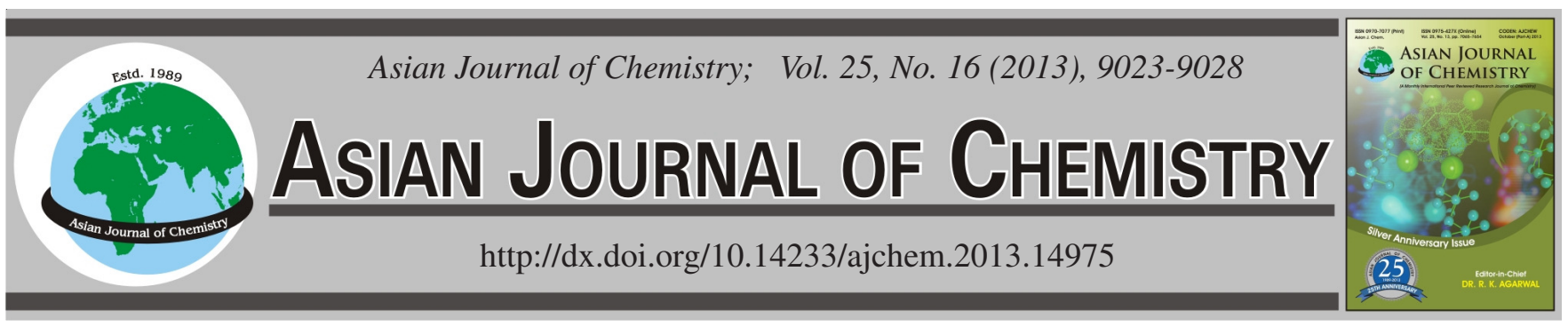

\title{
Ionic Liquid-Based Ultrasound-Assisted Dispersive Liquid-Liquid Microextraction Followed by High- Performance Liquid Chromatography for the Determination of Bisphenol-A in Sediment Samples
}

\author{
C. ZHANG ${ }^{1,2}$, X.Y. WAN ${ }^{1,2}$, C.Z. ZHOU ${ }^{1,2}$ and Y. $\mathrm{LI}^{1,2, *}$
}

${ }^{1}$ Resources and Environmental Research Academy, North China Electric Power University, Zhuxinzhuang, Dewai, Beijing 102206, P.R. China ${ }^{2}$ MOE Key Laboratory of Regional Energy Systems Optimization, North China Electric Power University, P.O. Box 58, Beijing 102206, P.R. China

*Corresponding author: Fax: +86 51971241; Tel: +86 51971241; E-mail: liyuxx@jlu.edu.cn

\begin{abstract}
An ionic liquid-based ultrasound-assisted dispersive liquid-liquid microextraction (IL-UAE-DLLME) method was developed for extraction of bisphenol-A from sediments. The analyte were determined by high-performance liquid chromatography equipped fluorescence (FLD) and ultraviolet (VWD) detector. The conditions of DLLME were optimized by an interactive orthogonal array design. Both of the extraction recovery (ER) and extraction factor (EF) were set as the responses for variance analysis. The optimum conditions were as follow: $0.9 \mathrm{~mL}$ of acetonitrile, $80 \mu \mathrm{L}$ of ion liquid, $7 \mathrm{~mL}$ of water sample, $5.5 \mathrm{of} \mathrm{pH}, 15 \%$ of salt effect and $<10 \mathrm{~s}$ of extraction time.
\end{abstract}

Key Words: Ionic liquid, Ultrasonic-assisted, Dispersive liquid-liquid microextraction, Bisphenol-A, Sediments.

\section{INTRODUCTION}

Bisphenol-A (BPA) was widely used as an monomer in the production of epoxy resins and polycarbonate plastics. The final productions of bisphenol-A were widely used as coatings on cans, plastic bottles, adhesives, artificial teeth and food packages $^{1,2}$. Bisphenol-A exhibited an estrogenic activity and carcinogenesis even at $\mu \mathrm{g} / \mathrm{L}$ levels and showed a high potential as endocrine disruptor interfering the endocrine system in human and wildlife ${ }^{3}$. European Union regarded the milk bottles containing bisphenol-A would induce the sexual precocity of babies. In 2011, the milk bottles with bisphenol-A have been forbidden to produce. Due to extensive application in chemical industry, large amount of bisphenol-A was discharged into the environment every year. From the year of 2006-2010, the global demand of bisphenol-A was predicted to increase from 3.9 to 5.0 million tonnes ${ }^{4}$. A majority of bisphenol-A was disposed into the aquatic environment mainly via the wastewater from plastics-producing industrial plants and landfill sites $^{5}$. Few of bisphenol-A released into the soil system and air via landfill leachate and handling process gas, respectively ${ }^{4,6}$. Hence, most of the bisphenol-A finally ended up in aquatic environment, such as river system. In river environment, the sediments played a critical role in monitoring the migration, fate and biogeochemistry of contaminant ${ }^{7}$. Moreover, the previous study suggested the sediment in riverine was a major place and a potential source of bisphenol- $\mathrm{A}^{8}$. Therefore, from the perspective of environmental risk to human exposed to bisphenol-A, a sensitive method was necessary for determining bisphenol-A at trace level in different sediment matrixes.

Recently, the quantity and damage of contaminants in aquatic environment had caught attentions of worldwide. However, the concentration of contaminant in natural environment was usually inferior to $\mathrm{mg} / \mathrm{L}$ or $\mu \mathrm{g} / \mathrm{L}$. Therefore, many pretreatment methods were developed to determine organic chemicals in different sediment samples. The preparation methods for organics in solid samples include Soxhlet extraction, ultrasonic extraction (UE), pressurized liquid extraction (PLE) ${ }^{9}$ and supercritical fluid extraction $(\mathrm{SFE})^{10}$. For the pretreatment of liquid sample, the following method were used, such as liquid-liquid extraction (LLE), solid-phase microextraction $(\mathrm{SPME})^{11}$, single drop microextraction (SDME) $)^{12}$, stir bar sorptive extraction $(\mathrm{SBSE})^{13}$ and hollow fiber liquid-phase microextraction (HF-LLME) ${ }^{14}$. These methods are oriented towards to free or consume a few micro-liters of organic solvent in the extraction process. However, they suffered from disadvantages as follows: the fiber of SPME was fragile and expensive; the single-drop of SDME was instability; SBSE required a long time for extraction; the extraction of HF-LLME was limited by the small surface of the fiber ${ }^{15}$. A rapid, high efficiency and simple-step pretreatment method was rather desirable.

A method called dispersive liquid-liquid microextraction (DLLME) was employed by Razaee ${ }^{16}$ for the extraction of organic compounds in water. The method based on the ternary 
component solvent system (extractant-dispersant-water sample), is a novel modality of LPME with advantages of simply operation, rapidity, low cost, high efficiency, environmental benefit and wide application prospects in trace analysis ${ }^{17}$.

At present, this method has been successfully applied for the determination of organic compounds and metal ions in liquid samples ${ }^{18}$. The previous studies reported that the DLLME were applied to determine organophosphorus pesticides (OPPs) in vegetables and tea ${ }^{19,20}$, polychlorinated biphenyls (PCBs) in soil ${ }^{21}$, multiple pesticide residues in soils and sulfonylurea herbicides in $\operatorname{soil}^{22}$. Besides, Li firstly introduced DLLMESFO to determinate decabrominated diphenyl ether (decaBDE) in sediment samples ${ }^{23}$. However, the application of UAE combined with DLLME for the determination of bisphenol-A at trace level in the riverine sediments was still scarce.

In the present work, ionic liquid based ultrasound-extraction dispersive liquid-liquid microextraction (IL-UAE-DLLME) combined with high-performance liquid chromatography was applied for the determination of bisphenol-A in environmental sediment samples. The analyte was firstly extracted from sediment to the organic-solvent by UAE and then the organicsolvent was used as the dispersant of DLLME. Additionally, an interactive orthogonal array design methodology was employed for the optimization of extraction conditions of DLLME. Meanwhile, the real sediment sample analysis, interference experiments and inter laboratory experiments were also been investigated.

\section{EXPERIMENTAL}

Bisphenol-A (99\%+, Aldrich, USA); 17 $\alpha$-ethynyl estrdiol, estrone, estradiol and estriol (99\%+, Sigma, USA); [C6MIM] [PF6] (99\%, Shanghai, China); Sodium Chloride (GR, Tianjin, China); acetonitrile (HPLC-grade, Honeywell Burdick \& Jackson, USA); acetone and methanol (HPLC-grade, J \& K Baker, USA); hydrochloride acid and sodium hydroxide (AR, Tianjin, China); Deionized water were prepared by using Direct-Q3UV ( Millipore, France).

Stock solution: Appropriate amount of bisphenol-A was weighted with high-precision electronic analytic balance (FA1004, Shanghai, China) and resolved into acetonitrile. 200 $\mathrm{mg} / \mathrm{L}$ of stock solution was prepared and stored at $4{ }^{\circ} \mathrm{C}$. This solution was stable for one week and diluted further to the required concentrations before use.

Sediment samples were collected from the Songhua river (Jilin, Jilin Province, China), the Yellow river (Jinan, Shandong Province, China) and the Liao river (Provincial boundary section of Liaoning and Jilin Province, China). The collected samples were air-dried and crushed to pass through $150 \mu \mathrm{m}$ sieve. $2 \mathrm{~g}$ sediment sample was exactly weighed into a $10 \mathrm{~mL}$ screw cap glass centrifuge tube and spiked with the standard solution by a $50 \mathrm{~mL}$ micro-syringe. The spiked samples were located in a darkened fume hood for $15 \mathrm{~h}$ and the solution was completely evaporated and sorption-desorption reached equilibrium.

General procedure: $2 \mathrm{~g}$ of sediment sample was ultrasonic-assistant extraction with $4 \mathrm{~mL}$ acetonitrile for $1 \mathrm{~h}$ and then centrifuged at $4000 \mathrm{rpm}$ for $7 \mathrm{~min}$ (refrigerated centrifuge 2-16PK, Sigma, Germany). The supernatant was filtered through a $0.2 \mu \mathrm{m}$ nylon syringe filter (Tianjing, China) and filtrate was used as dispersive solvent in the DLLME step.

$7 \mathrm{~mL}$ of $0.15 \mathrm{~g} / \mathrm{mL} \mathrm{NaCl}$ solution was placed in a $10 \mathrm{~mL}$ screw cap glass centrifuge tube with a conical bottom. $80 \mu \mathrm{L}$ of [C6MIM][PF6] (ionic liquid) was added into $0.9 \mathrm{~mL}$ of acetonitrile (containing bisphenol-A) used as the extraction solvent. Then, mixed solution was transferred to the centrifuge tube rapidly. The mixture was gently shaken by hands for several seconds. After centrifugation for $10 \mathrm{~min}$ at $5000 \mathrm{rpm}$, ionic liquid phase sank at the bottom of the centrifuge tube. $10 \mu \mathrm{L}$ of sediment phase was removed using a $50 \mu \mathrm{L}$ microsyringe (Agilent, USA) and injected into the HPLC system for analysis.

Detection method: Agilent 1200 HPLC (Agilent, USA) was equipped with $20 \mu \mathrm{L}$-loop manual injector, a vacuum degasser (G1322A), a double pump (G1312A), a varied wavelength detector (G1314B, Agilent, USA) set at $280 \mathrm{~nm}$ and a fluorescence detector (G1321A, Agilent, USA) set at excitation and emission wavelengths of 230 and $315 \mathrm{~nm}$, respectively. Data collection, integration and analysis were accomplished by the Agilent ChemStation software. The analytical chromatographic column was ZORBAX SB-C ${ }_{18}(250 \mathrm{~mm} \times 4.6 \mathrm{~mm}$, $5 \mu \mathrm{m}$, Agilent).

Gradient elution was used to separate the target compound with other compounds. The gradient program was as follows: 0-38 min methanol: demineralized water $=50 \%: 50 \%(\mathrm{v}: \mathrm{v})$, 38-39 min the methanol increased from 50-100 \%, hold on for $65 \mathrm{~min}$. The retention time of bisphenol-A was found to be $29.7 \mathrm{~min}$. The column temperature was kept at $30^{\circ} \mathrm{C}$ and the flow rate was $1 \mathrm{~mL} / \mathrm{min}$.

\section{RESULTS AND DISCUSSION}

Selection of extraction solvent in UAE/dispersive solvent in DLLME: In this study, the solvent was used as extraction solvent in the UAE step and then as the dispersive solvent in the DLLME step. Therefore, the selection of solvent was based on not only extraction efficiency from sediment but also miscibility in ionic liquid and aqueous phase. For this purpose, acetonitrile, methanol and acetone were tested and optimized in two steps extraction. As shown in Fig. 1, acetonitrile had the highest extraction recovery. Therefore, acetonitrile was selected as extraction solvent for further experiments.

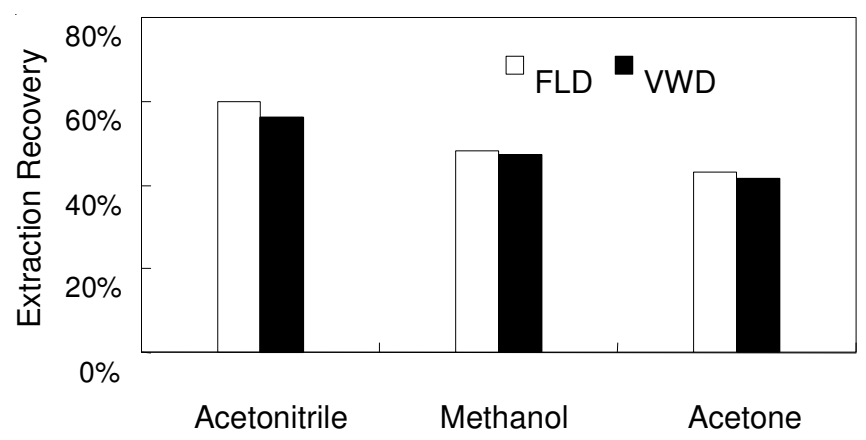

Fig. 1. Effect of dispersive solvent on extraction efficiency. Extraction conditions of DLLME: water sample volume, $5 \mathrm{~mL}$; ionic liquid volume, $60 \mu \mathrm{L}$; dispersive solvent volume, $0.4 \mathrm{~mL}$; spiked level, $0.8 \mu \mathrm{g} / \mathrm{g}$; $\mathrm{pH} 5.5$; without addition of $\mathrm{NaCl}$; extraction time, $<10 \mathrm{~s}$ 
Interactive orthogonal array design of DLLME: $\mathrm{A} \mathrm{L}_{16}$ $\left(2^{15}\right)$ interactive orthogonal array design was employed for screening significant factors affecting the DLLME process. The volume of acetonitrile (A), ionic liquid volume (B), volume of water sample (C), $\mathrm{pH}(\mathrm{D})$, salt effect (E) and extraction time (F) were set as variables with high and low levels (Table-1). Both of extraction recovery (ER) and enrichment factor (EF) were set as the responses for the variance analysis. All the experimental trials were conducted in duplicate. The results of variance analysis indicated that the volume of acetonitrile and ionic liquid volume were statistically significant for EF (showed in Table-2), while the salt effect was significant for ER as evidenced by $F$ value $>F_{0.005}$. Thus, the volume of acetonitrile, ionic liquid volume and salt effect were needed to be further optimized by the single factors test. In addition, the other factors and the interaction effect investigated performed less significant effects on the extraction procedure based on F value $\left(<\mathrm{F}_{0.005}\right)$. Therefore, under consideration of higher extraction efficiency and simplicity of procedure, $\mathrm{pH}$, the volume of aqueous phase and extraction time were selected as $5.5,7 \mathrm{~mL}$ and less than $10 \mathrm{~s}$, respectively.

TABLE-1

LEVELS OF THE EXTRACTION FACTORS FOR DLLME BY INTERACTIVE ORTHOGONAL ARRAY DESIGN

\begin{tabular}{clcc}
\hline Symbols & \multicolumn{1}{c}{ Factors } & Low level & High level \\
\hline $\mathrm{A}$ & Volume of acetonitrile $(\mathrm{mL})$ & 0.4 & 0.7 \\
$\mathrm{~B}$ & Ionic liquid volume $(\mu \mathrm{L})$ & 50 & 70 \\
$\mathrm{C}$ & Volume of water sample $(\mathrm{mL})$ & 5 & 7 \\
$\mathrm{D}$ & $\mathrm{pH}$ & 5.5 & 7 \\
$\mathrm{E}$ & Salt effect $(\mathrm{w} / \mathrm{v})$ & 0 & $10 \%$ \\
$\mathrm{~F}$ & Extraction time & $<10 \mathrm{~s}$ & $20 \mathrm{~min}$ \\
\hline
\end{tabular}

\section{One variable at a time design for DLLME}

Salt effect: In this experiment, salt effect was tested in the range of $0-20 \%(\mathrm{w} / \mathrm{v})$. Without addition of salts, the sediment phase volume was not enough for $10 \mu \mathrm{L}$ HPLC injection; while, at the $20 \%$, the density of extraction phase probably approached or even exceeded sodium chloride solution, so that dispersed tiny droplets of ionic liquid cannot sink through centrifugation. By increasing the salt effect from 5 to $15 \%$, the extraction recovery increased obviously and the enrichment factor remained stable, as shown in Fig. 2. Meanwhile, the

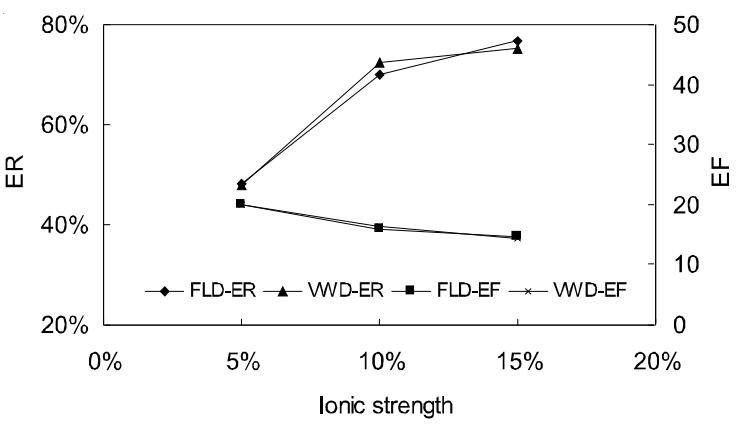

Fig. 2. Effect of salt effect on extraction efficiency. Extraction conditions of DLLME: water sample volume, $7 \mathrm{~mL}$; ionic liquid volume, 50 $\mu \mathrm{L}$; acetonitrile volume, $0.7 \mathrm{~mL}$; concentration of bisphenol-A in acetonitrile, $0.4 \mathrm{mg} / \mathrm{L}$; $\mathrm{pH} 5.5$; extraction time, $<10 \mathrm{~s}$

sediment phase increase from 17 to $37 \mu \mathrm{L}$, which can be explained that increase of $\mathrm{NaCl}$ resulted in the decreasing of solubility of the extraction solvent in aqueous phase. Based on the analysis, all the subsequent experiments are carried out with the addition of $15 \% \mathrm{NaCl}(\mathrm{w} / \mathrm{v})$.

Effect of volume of extraction solvent and dispersive solvent: The effect of ionic liquid (extraction solvent) volume on the extraction efficiency was tested in the range of $30-80 \mu \mathrm{L}$. As shown in Fig. 3, with the increasing of extraction solvent volume, the extraction recovery continually rose to more than $90 \%$, while the enrichment factor was kept within an order of magnitude. Thus, $80 \mu \mathrm{L}$ of ionic liquid was chosen as optimum volume.

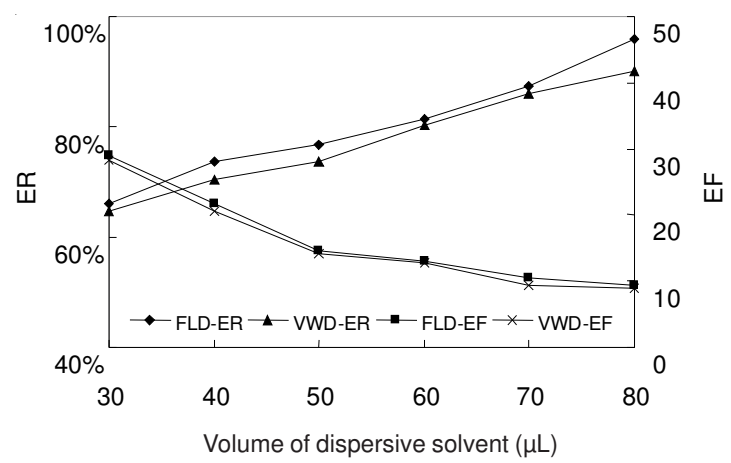

Fig. 3. Effect of dispersive solvent volume on extraction efficiency. Extraction conditions of DLLME: water sample volume, $7 \mathrm{~mL}$; acetonitrile volume, $0.7 \mathrm{~mL}$; concentration of bisphenol-A in acetonitrile, $0.4 \mathrm{mg} / \mathrm{L}$; $\mathrm{pH} 5.5$; concentration of $\mathrm{NaCl}, 15 \%$ (w/v); extraction time, $<10 \mathrm{~s}$

\begin{tabular}{|c|c|c|c|c|c|c|c|c|c|c|}
\hline \multirow{3}{*}{ Source } & \multicolumn{9}{|c|}{$\begin{array}{c}\text { TABLE-2 } \\
\text { IS BASED ON ER AND EF FOR ORTHOGONA }\end{array}$} & \multirow{3}{*}{ Significance } \\
\hline & \multicolumn{2}{|c|}{ Sum of squares } & \multirow{2}{*}{$\begin{array}{l}\text { Degree of } \\
\text { freedom }\end{array}$} & \multicolumn{2}{|c|}{ Mean square } & \multicolumn{2}{|c|}{ F-ratio } & \multicolumn{2}{|c|}{ Critical value } & \\
\hline & $\mathrm{ER}^{\mathrm{a}}$ & $\mathrm{EF}^{\mathrm{b}}$ & & ER & $\mathrm{EF}$ & ER & $\mathrm{EF}$ & 0.01 & 0.05 & \\
\hline $\mathrm{A}$ & 0.0215 & 102.0387 & 1 & 0.0215 & 102.0387 & 3.5024 & 141.8954 & 22.78 & 16.26 & $* *$ \\
\hline B & 0.0351 & 72.9617 & 1 & 0.0351 & 72.9617 & 5.7184 & 101.4608 & 22.78 & 16.26 & $* *$ \\
\hline $\mathrm{C}$ & 0.0143 & 15.3815 & 1 & 0.0143 & 15.3815 & 2.3265 & 21.3895 & 22.78 & 16.26 & - \\
\hline $\mathrm{D}$ & 0.0039 & 0.8765 & 1 & 0.0039 & 0.8765 & 0.6346 & 1.2188 & 22.78 & 16.26 & - \\
\hline $\mathrm{E}$ & 0.1410 & 3.3235 & 1 & 0.1410 & 3.3235 & 22.9693 & 4.6217 & 22.78 & 16.26 & $* *$ \\
\hline F & 0.0082 & 0.2881 & 1 & 0.0082 & 0.2881 & 1.3340 & 0.4006 & 22.78 & 16.26 & - \\
\hline $\mathrm{A} \times \mathrm{B}^{\mathrm{c}}$ & 0.0038 & 5.9107 & 1 & 0.0038 & 5.9107 & 0.6237 & 8.2195 & 22.78 & 16.26 & - \\
\hline $\mathrm{A} \times \mathrm{C}$ & 0.0001 & 2.8351 & 1 & 0.0001 & 2.8351 & 0.0240 & 3.9425 & 22.78 & 16.26 & - \\
\hline $\mathrm{B} \times \mathrm{C}$ & 0.0002 & 0.0015 & 1 & 0.0002 & 0.0015 & 0.0258 & 0.0021 & 22.78 & 16.26 & - \\
\hline $\mathrm{B} \times \mathrm{E}$ & 0.0166 & 0.9698 & 1 & 0.0166 & 0.9698 & 2.7047 & 1.3486 & 22.78 & 16.26 & - \\
\hline$e^{d}$ & 0.0307 & 3.5956 & 5 & 0.0061 & 0.7191 & - & - & - & - & - \\
\hline$T^{f}$ & 0.2755 & 208.1826 & 15 & - & - & - & - & - & - & - \\
\hline
\end{tabular}

${ }^{\mathrm{a}} \mathrm{ER}, \%=$ Extraction recovery; ${ }^{\mathrm{b}} \mathrm{EF}=$ Extraction factor; ${ }^{\mathrm{c}} \mathrm{The}$ " $\mathrm{x}$ " means interaction between two factors; ${ }^{\mathrm{d}}$ Error; ${ }^{\mathrm{f}}$ The total of deviations. 
In order to improve the extraction recovery and extraction factor, the experiment increased the volume of dispersive solvent from 0.7 to $0.9 \mathrm{~mL}$. The result showed that the extraction factor increased from 10 to 12 , while the extraction recovery kept stable. Thereby, $0.9 \mathrm{~mL}$ of acetonitrile was chosen as the volume of dispersive solvent in order to obtain the highest extraction factor. However, as the volume of dispersive solvent exceeded $1 \mathrm{~mL}$, the dispersed tiny droplets of ionic liquid can't sink, possibly due to the lower density than sodium chloride solution by adding the acetonitrile.

\section{Analytical performance of HPLC-VWD and HPLC-FLD methods}

Linearity and detection limits: The detection limits and linear ranges were important parameters to evaluate the sensitivity of an analytical method. Under the optimum condition obtained, linearity was observed in the range of $0.05-10 \mu \mathrm{g} / \mathrm{g}$ for FLD and 0.5-50 $\mu \mathrm{g} / \mathrm{g}$ for VWD with the correlation coefficient $\left(\mathrm{R}^{2}\right)$ of 0.9999 . Limits of detection (LOD) for FLD and VWD, on the basis of three times of signal to noise ratio ( $\mathrm{S} / \mathrm{N}$ $=3$ ), were 0.21 and $28.6 \mathrm{ng} / \mathrm{g}$, respectively. From the data above, it was clear that LOD for FLD was two orders of magnitude lower than that for VWD, implying that FLD method was more sensitive for determination of the trace bisphenol-A in sediment.

Real sediment sample analysis: The applicability and reliability of FLD and VWD methods were evaluated by analyzing bisphenol-A in sediment samples, collected at Songhua river, Yellow river and Liao river in China. The sediment samples were pretreated as described above, first extracted by acetonitrile, concentrated with the DLLME method and analyzed by HPLC-FLD/VWD. The relative recoveries and standard deviations of bisphenol-A in spiked sediment were investigated (Table-3).

The results indicated that the sediment samples of the Songhua river and the Yellow river were free of bisphenol-A. The bisphenol-A concentration in the Liao river was determined to be $0.2557 \mu \mathrm{g} / \mathrm{g}$ by HPLC-FLD, but lower than the quantization limit of HPLC-VWD. In addition, the sediment samples from the Songhua river, the Yellow river and the Liao river were spiked at the different levels of bisphenol-A to assess matrix effects. As seen in Table-3, the recovery ranged 90.77$110.59 \%$ with the RSD of 2.87-7.02\% for HPLC-FLD method and 86.41-102.07 \% with 1.68-8.72\% for HPLC-VWD method. Figs. 4 and 5 showed chromatograms of blank sediment sample and sample spiked with $0.5 \mu \mathrm{g} / \mathrm{g}$ of bisphenol-A in Liao river, respectively. The results demonstrated that the three real sediment matrices had little effect on the HPLCFLD and HPLC-VWD methods.

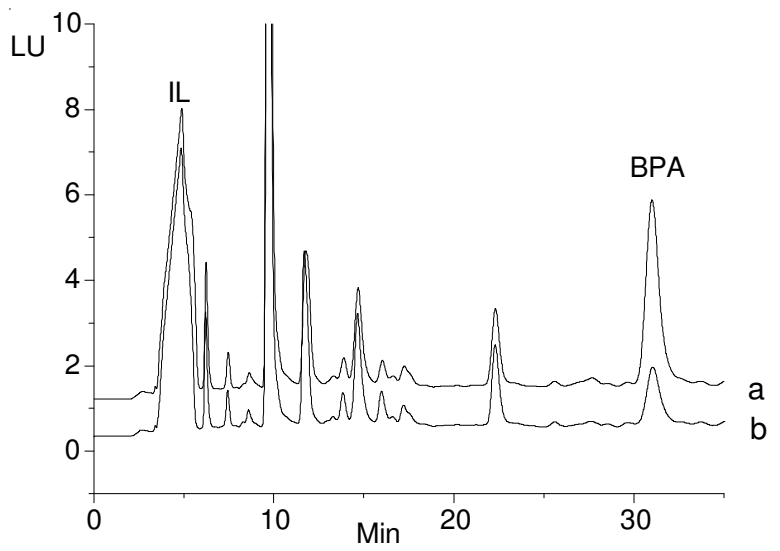

Fig. 4. HPLC-FLD chromatograms of blank sediment sample (b) and sample spiked with $0.5 \mu \mathrm{g} / \mathrm{g}$ of bisphenol-A (a) in Liao river

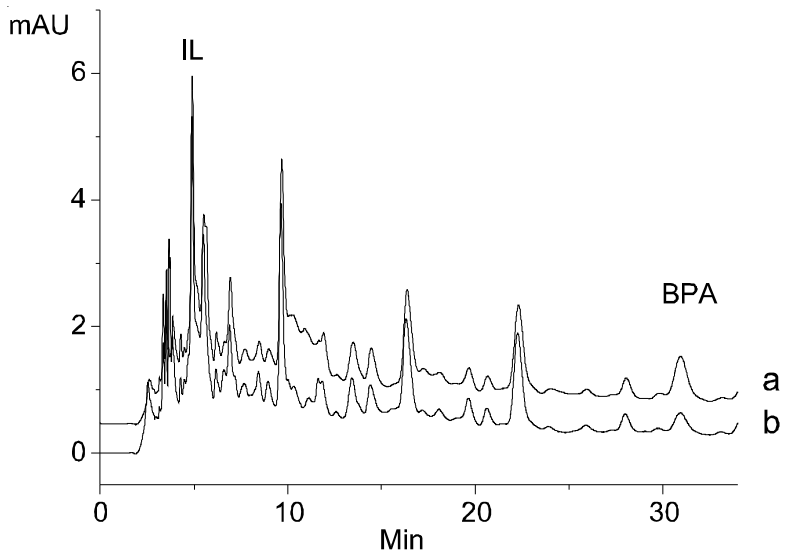

Fig. 5. HPLC-VWD chromatograms of blank sediment sample (b) and sample spiked with $0.5 \mu \mathrm{g} / \mathrm{g}$ of bisphenol-A (a) in Liao river

TABLE-3

RELATIVE RECOVERIES AND STANDARD DEVIATIONS OF BPA FROM SPIKED SEDIMENT SAMPLES

\begin{tabular}{|c|c|c|c|c|c|c|c|}
\hline & \multicolumn{4}{|c|}{ HPLC-FLD } & \multicolumn{3}{|c|}{ HPLC-VWD } \\
\hline & $\begin{array}{l}\text { Added } \\
(\mu \mathrm{g} / \mathrm{g})\end{array}$ & $\begin{array}{l}\text { Found } \\
(\mu \mathrm{g} / \mathrm{g})\end{array}$ & $\begin{array}{c}\text { Relative recovery } \\
(\%)\end{array}$ & $\begin{array}{c}\operatorname{RSD}^{\mathrm{a}}(\% \\
\mathrm{n}=3)\end{array}$ & $\begin{array}{l}\text { Found } \\
(\mu \mathrm{g} / \mathrm{g})\end{array}$ & $\begin{array}{l}\text { Relative recovery } \\
(\%)\end{array}$ & $\begin{array}{c}\mathrm{RSD}^{\mathrm{a}} \\
(\%, \mathrm{n}=3)\end{array}$ \\
\hline \multirow{5}{*}{ Songhua river } & 0 & $\mathrm{nd}^{\mathrm{b}}$ & - & - & nd & - & - \\
\hline & 0.05 & 0.0553 & 110.59 & 6.03 & - & - & - \\
\hline & 0.5 & 0.5237 & 104.7 & 4.39 & 0.4767 & 95.34 & 2.10 \\
\hline & 1 & 0.9995 & 99.95 & 3.07 & 5.1035 & 102.07 & 3.95 \\
\hline & 10 & 10.1364 & 101.36 & 3.94 & 10.1364 & 101.36 & 3.94 \\
\hline \multirow{4}{*}{ Yellow river } & 0 & nd & - & - & nd & - & - \\
\hline & 0.05 & 0.0520 & 104.06 & 4.56 & - & - & - \\
\hline & 0.5 & 0.4767 & 95.34 & 2.10 & 0.4977 & 99.53 & 1.86 \\
\hline & 5 & 5.1035 & 102.07 & 3.95 & 4.9789 & 99.58 & 2.99 \\
\hline \multirow{4}{*}{ Liao river } & 0 & 0.2557 & - & 5.03 & nd & - & - \\
\hline & 0.05 & 0.0454 & 90.77 & 7.02 & - & - & - \\
\hline & 0.5 & 0.5113 & 102.25 & 5.10 & 0.5092 & 101.85 & 8.72 \\
\hline & 5 & 4.5613 & 91.23 & 2.87 & 4.3206 & 86.41 & 1.68 \\
\hline
\end{tabular}

${ }^{\mathrm{a}} \mathrm{RSD}, \%$ relative standard deviation, $\mathrm{n}=3 .{ }^{\mathrm{b}}$ nd, not detected. 
Interference experiment: Interference experiment was used to examine whether other endocrine disrupting chemicals (EDCs) disturbed the determination of bisphenol-A using the proposed methods. As shown in Fig. 6, it was obvious that bisphenol-A was separated from other EDCs involving $17 \alpha-$ ethynyl estrdiol (EE2), estrone (E1), estradiol (E2) and estriol (E3) for the HPLC-FLD and HPLC-VWD methods. Moreover, the four EDCs mentioned above can be separated from each other, although the E1 had little response for HPLC-FLD method. The results implied that the selected EDCs in sediment samples could be determined simultaneously.

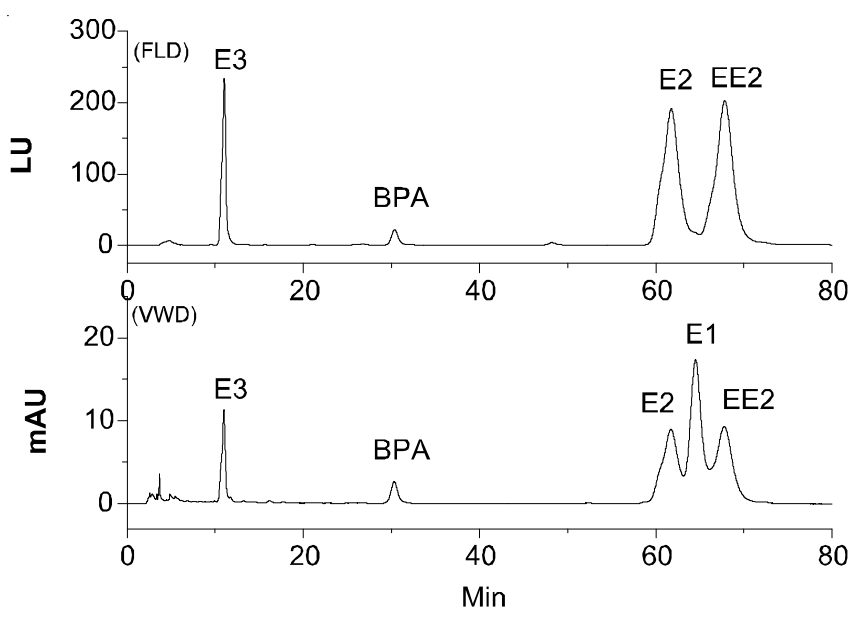

Fig. 6. HPLC-FLD/VWD chromatograms of bisphenol-A and other four EDCs under the optimum chromatographic condition obtained

Interlaboratory comparative experiment: Interlaboratory comparative experiment was performed to validate the repeatability of HPLC-FLD and HPLC-VWD methods at spiking level of $10 \mu \mathrm{g} / \mathrm{g}$. Found 1 and found 2 were determined by
Agilent 1200 HPLC in laboratory of North China Electric Power University and by Summit HPLC in laboratory of Liaoning Environment Monitoring Centre, respectively. The analysis results were showed in Table-4.

Furthermore, F-test and T-test were employed to analyze the inter-laboratory results of HPLC-FLD and HPLC-VWD methods. The results of F-test were as followed: $F=2.00, F_{0.05}$ $=9.6, \mathrm{~F}<\mathrm{F}_{0.05}$ (FLD method) $\mathrm{F}=1.92, \mathrm{~F}_{0.05}=9.6, \mathrm{~F}<\mathrm{F}_{0.05}$ (VWD method). The results of T-test were as followed: $\mathrm{t}=$ $0.19, \mathrm{t}_{0.05,8}=2.31, \mathrm{t}<\mathrm{t}_{0.05,10}$ (FLD method); $\mathrm{t}=0.33, \mathrm{t}_{0.05,8}=$ $2.31, \mathrm{t}<\mathrm{t}_{0.05,10}$ (VWD method). The results indicated that there were no significant differences between laboratories in precision and accuracy.

Comparison of DLLME with other methods: The proposed HPLC-FLD and HPLC-VWD methods were compared with relevant reported methods, such as Tween 80 extraction-SPEHPLC-VWD ${ }^{24}$, MAE-GC-MS ${ }^{25}$, ultrasonic-GC-MS ${ }^{26}$ and Soxhlet-HPLC-MS ${ }^{27}$, in terms of LOD, RSD, volume of organic solvent, extraction time and sample consumption (Table-5). The results showed that LOD of proposed FLD method was at the same order of magnitude as soxhlet-HPLC-MS and much lower than other methods. Especially, the proposed methods not only use the less volume of organic solvent and the sample, but also meet the demand of the high sensitive, great enrichment factors and good yields in a simple and fast way.

\section{Conclusion}

This paper developed two analytical methods of DLLME followed by HPLC with fluorescence or ultraviolet detection for the determination of bisphenol-A in sediment. The results demonstrated that the proposed methods performed a satisfied repeatability (1.68-8.72\%), high relative recoveries (86.41$110.59 \%)$, wide linear range $(0.05-10 \mu \mathrm{g} / \mathrm{g}$ for HPLC-FLD

\begin{tabular}{|c|c|c|c|c|}
\hline \multicolumn{5}{|c|}{$\begin{array}{c}\text { TABLE-4 } \\
\text { PRECISION AND ACCURACY BETWEEN TWO LABORATORIES }\end{array}$} \\
\hline \multirow{2}{*}{ Run number } & \multicolumn{2}{|c|}{ HPLC-FLD } & \multicolumn{2}{|c|}{ HPLC-VWD } \\
\hline & Found $1(\mu \mathrm{g} / \mathrm{g})$ & Found $2(\mu \mathrm{g} / \mathrm{g})$ & Found $1(\mu \mathrm{g} / \mathrm{g})$ & Found $2(\mu \mathrm{g} / \mathrm{g})$ \\
\hline 1 & 9.82 & 8.61 & 9.27 & 9.63 \\
\hline 2 & 10.00 & 10.73 & 9.46 & 8.95 \\
\hline 3 & 8.99 & 8.94 & 10.06 & 10.31 \\
\hline 4 & 10.59 & 9.22 & 8.70 & 10.55 \\
\hline 5 & 9.73 & 9.47 & 9.36 & 10.42 \\
\hline $\mathrm{SD}^{\mathrm{a}}(\mu \mathrm{g} / \mathrm{g})$ & 0.57 & 0.81 & 0.49 & 0.67 \\
\hline $\operatorname{RSD}^{\mathrm{b}}(\%)$ & 5.85 & 8.65 & 5.18 & 6.74 \\
\hline SD of reproductivity $(\mu \mathrm{g} / \mathrm{g})$ & \multicolumn{2}{|c|}{0.31} & \multicolumn{2}{|c|}{0.41} \\
\hline RSD of reproductivity (\%) & \multicolumn{2}{|c|}{3.23} & \multicolumn{2}{|c|}{4.24} \\
\hline Recovery $(\%)$ & \multicolumn{2}{|c|}{93.10} & \multicolumn{2}{|c|}{96.71} \\
\hline
\end{tabular}

TABLE-5

COMPARISON OF ULTRASONIC-DLLME-HPLC-FLD/VWD WITH OTHER EXTRACTION METHODS FOR DETERMINATION OF BPA

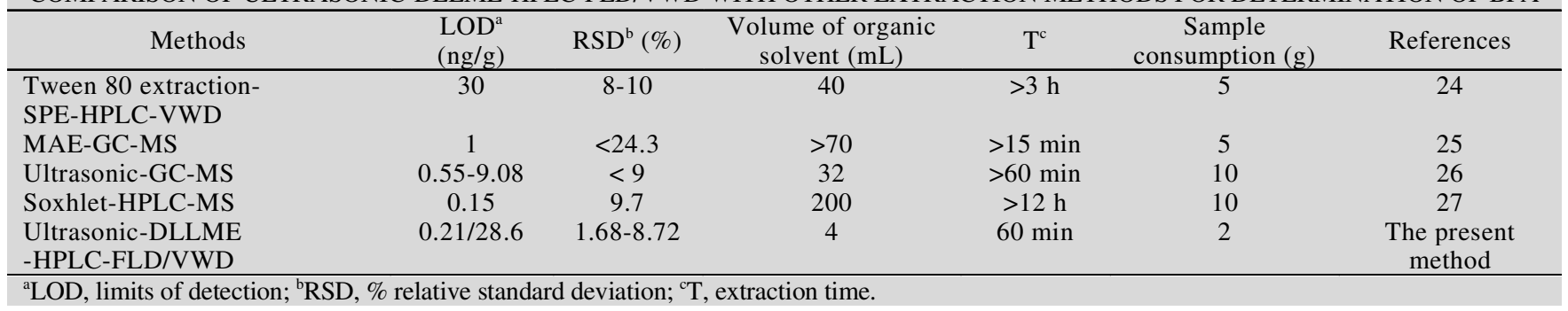


and $0.5-50 \mu \mathrm{g} / \mathrm{g}$ for HPLC-VWD) and detection limits of $\mathrm{ng} / \mathrm{g}$ level. Besides, the proposed methods can be applied for determination of bisphenol-A in real sediment samples. The bisphenol-A could be preferably separated from the other endocrine disrupting compounds (E1, E2, EE2 and E3) in interference experiments. The inter-laboratory comparative experiment showed that the HPLC-FLD and HPLC-VWD methods had acceptable repeatability. Compared with other extraction methods, the proposed methods had the advantages of high sensitivity, environmental benefit, the low cost of equipments and simple operation. The proposed methods could be applied for analyzing compounds in solid environmental samples.

\section{ACKNOWLEDGEMENTS}

Financial support was provided by the Ministry of Science and Technology China ('973' Project No. 2004CB3418501).

\section{REFERENCES}

1. M. Umar, F. Roddick, L.H. Fan and HA. Aziz, Chemosphere, 90, 2197 (2013).

2. H. Fromme, T. Küchler, T. Otto, K. Pilz, J. Müller and A. Wenzel, Water Res., 36, 1429 (2002).

3. S. Flint, T. Markle, S. Thompson and E. Wallace, J. Environ. Manage., 104, 19 (2012).

4. Y.Q. Huang, C.K.C. Wong, J.S. Zheng, H. Bouwman, R. Barra, B. Wahlström, L. Neretin and M.H. Wong, Environ. Int., 42, 91 (2012).

5. D.P. Mohapatra, S.K. Brar, R.D. Tyagi and R.Y. Suramapalli, Chemosphere, 78, 923 (2010).

6. X. Wang, H.L. Zeng, L.X. Zhao and J.M. Lin, Anal. Chim. Acta, 556, 313 (2006).
7. Y. Li, F. Yang, D.M. Dong, Y.Z. Lu and S.H. Guo, Chemosphere, 62, 1709 (2006).

8. M. Peck, R.W. Gibson, A. Kortenkamp and E.M. Hill, Environ. Toxicol. Chem., 23, 945 (2004).

9. A. Mustafa and C. Turner, Anal. Chim. Acta, 703, 8 (2011).

10. H. Sovová, J. Supercrit. Fluid, 66, 73 (2012).

11. H. Lord and J. Pawliszyn, J. Chromatogr. A, 885, 153 (2000).

12. A. Jain and K.K. Verma, Anal. Chim. Acta, 706, 37 (2011).

13. F. David and P. Sandra, J. Chromatogr. A, 1152, 54 (2007).

14. H.M. Jiang, B. Hu, B.B. Chen and W.Q. Zu, Spectrochim. Acta B, 63, 770 (2008).

15. L.Y. Fu, X.J. Liu, J. Hu, X.N. Zhao, H.L. Wang and X.D. Wang, Anal. Chim. Acta, 632, 289 (2009).

16. M. Rezaee, Y. Assadi, M.R. Milani Hosseini, E. Aghaee, F. Ahmadi and S. Berijani, J. Chromatogr. A, 1116, 1 (2006).

17. X.H. Zang, Q.H. Wu, M.Y. Zhang, G.H. Xi and Z. Wang, Chin. J. Anal. Chem., 37, 161 (2009).

18. M. Rezaee, Y. Yamini and M. Faraji, J. Chromatogr. A, 1217, 2342 (2010).

19. E.C. Zhao, W.T. Zhao, L.J. Han, S.R. Jiang and Z.Q. Zhou, J. Chromatogr. A, 1175, 137 (2007).

20. S. Moinfar and M.R. Milani Hosseini, J. Hazard. Mater, 169, 907 (2009).

21. J. Hu, L.Y. Fu, X.N. Zhao, X.J. Liu, H.L. Wang, X.D. Wang and L.Y. Dai, Anal. Chim. Acta, 640, 100 (2009).

22. Q.H. Wu, C. Wang, Z.M. Liu, C.X. Wu, X. Zeng, J.L. Wen and Z. Wang, J. Chromatogr. A, 1216, 5504 (2009).

23. Y.H. Jian, Y. Hu, T. Wang, J.L. Liu, C. Zhang and Y. Li, Chin. J. Anal. Chem., 38, 62 (2010).

24. L. Patrolecco, S. Capri, S. De Angelis, S. Polesello, S. Valsecchi, J. Chromatogr. A, 1022, 1 (2004).

25. R. Liu, J.L. Zhou and A. Wilding, J. Chromatogr. A, 1038, 19 (2004).

26. J. Xu, L.S. Wu, W.Q. Chen and A.C. Chang, J. Chromatogr. A, 1202, 189 (2008).

27. S.G. Chu, H.G. Douglas and R.J. Letcher, J. Chromatogr. A, 1097, 25 (2005). 